\section{Peripheral pulmonary adenoid cystic carcinoma with substantial submucosal extension to the
proximal bronchus}

\author{
Hiromasa Inoue, Akinori Iwashita, \\ Hideaki Kanegae, Kazuyuki Higuchi, \\ Yutaka Fujinaga, Isao Matsumoto
}

\begin{abstract}
A rare case of a peripheral pulmonary adenoid cystic carcinoma is presented. Histological examination after lobectomy showed pronounced submucosal invasion of the carcinoma to proximal bronchi.
\end{abstract}

\section{Department of \\ Pathology \\ A Iwashita \\ H Kanegae \\ K Higuchi}

Department of

Surgery, Matsuyama Red Cross Hospital, Matsuyama, Japan

Y Fujinaga

I Matsumoto

Accepted 12 September 1990

Reprint requests to: Dr Inoue, Research Institute for Disease of the Chest, Faculty of Medicine, Kyushu University, 3-1-1 Maidashi, Higashiku, Fukuoka 812, Japan.

Histological appearance of the resected lung specimen: adenoid cystic carcinoma is covered with bronchial mucosa, infiltrating the bronchial wall.

(Haematoxylin and eosin.)

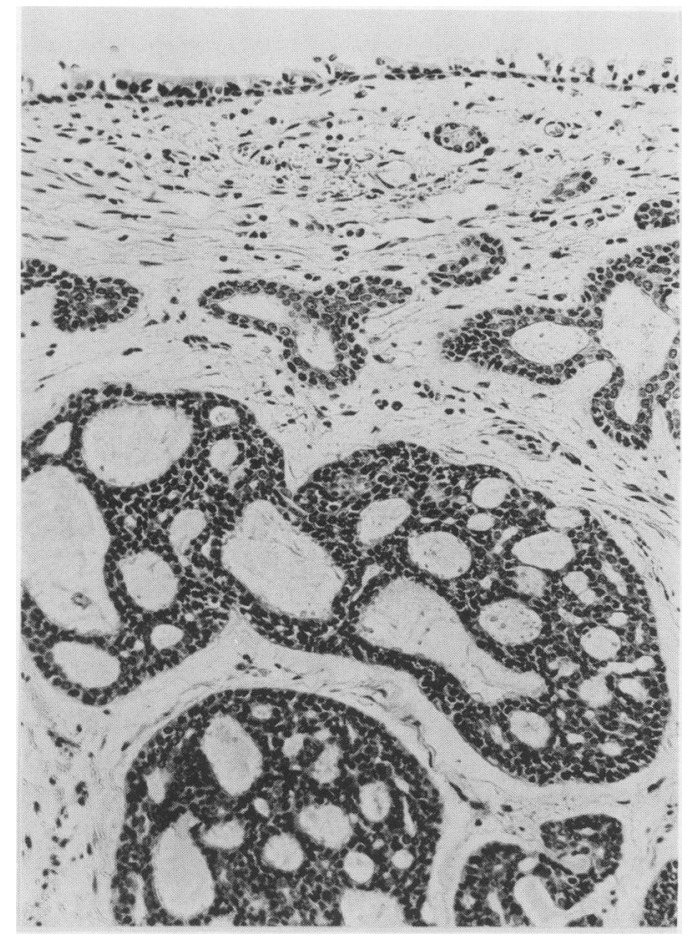

\section{Case report}

A 76 year old man presented in March 1987 with a two month history of non-productive cough. He had had pulmonary tuberculosis 30 years previously and had smoked two packs of cigarettes daily for more than $\mathbf{4 0}$ years.

On examination the patient was found to be mildly hypertensive, afebrile, and in no respiratory distress. There was no lymphadenopathy, and no evidence of tumour of the upper respiratory tract or salivary glands. The remainder of the examination, including that of the chest, showed nothing remarkable. The erythrocyte sedimentation rate was $44 \mathrm{~mm}$ in the first hour, the blood count was normal, and coagulation studies gave normal results. Pulmonary function tests showed that forced vital capacity (FVC) was 1.931 and forced expiratory volume in one second $\left(\mathrm{FEV}_{1}\right)$ $1 \cdot 131$. Arterial blood gas values when he was breathing room air were: oxygen tension $11.4 \mathrm{kPa}$, carbon dioxide tension $5.6 \mathrm{kPa}$, and $\mathrm{pH}$ 7.38. Microbiological and cytological investigations of the sputum were negative.

The chest radiographs showed two nodular uncalcified shadows, $4 \times 3 \mathrm{~cm}$ and $2 \times 1 \mathrm{~cm}$ in the posterior segment of the right upper lobe. Fibreoptic bronchoscopy showed normal appearances. Transbronchial biopsy under fluoroscopic guidance of the nodular shadow revealed adenoid cystic carcinoma. A computed tomogram of the chest did not show transbronchial wall thickening or hilar or mediastinal lymphadenopathy. There were no lesions in any other organs. A right upper lobectomy, including dissection of the hilar lymph nodes, was performed.

The operative specimen showed two masses in the posterior segment of the right upper lobe. The larger mass $(3 \mathrm{~cm}$ diameter) was elastic and soft, and communicated with the subsegmental bronchus. The cut surface was greyish white, and the margin was blurred. The distance from this larger mass to the proximal bronchial resection edge was $4 \mathrm{~cm}$. The smaller mass ( $1 \mathrm{~cm}$ diameter) in the apical subpleural region was hard. A frozen section was not obtained during thoracotomy.

The histological findings of the larger mass showed a characteristic cribiform pattern, composed of small cells with round hyperchromatic nuclei, and duct like structures containing material staining with periodic acid-Schiff, compatible with adenoid cystic carcinoma. Submucosal infiltrative spread of the tumour proximally from the peripheral mass was observed (figure). All the removed lymph nodes were tumour free. The bronchial stump was invaded by adenoid cystic carcinoma. The smaller mass was a tuberculoma.

Postoperative radiotherapy was not performed because of a persistent air leak.

\section{Discussion}

This is the first reported case of a peripheral pulmonary adenoid cystic carcinoma showing substantial submucosal extension to the proximal bronchus. The tumour usually arises in the trachea or central bronchi, unless it 
represents metastasis from another site. ${ }^{34}$ Occasional cases of peripheral adenoid cystic carcinoma, however, have been reported. Our review of published reports suggest that about $10 \%$ of primary pulmonary adenoid cystic carcinomas arise in peripheral lung. ${ }^{367}$ Most peripheral pulmonary adenoid cystic carcinomas situated in the main or lobar bronchi grow into the airway lumen in addition to growing beneath the bronchial epithelium. ${ }^{36}$ In this case, however, there was no polypoid mass or airway narrowing. The tumour was more prominent in the bronchial wall submucosa around the main tumour mass, in the posterior segment of the right upper lobe. These factors indicate that this was a peripheral tumour with submucosal extension to the proximal bronchus. The fact that the posterior segment of the right upper lobe is relatively small may promote submucosal invasion to the central bronchus.

Adenoid cystic carcinomas arising from the trachea or bronchus are associated with a high incidence of submucosal extension, and a tendency to recur locally if not excised completely. ${ }^{56}$ Such extension has not so far been described in the peripheral tumours. Because there were no signs of proximal bronchial wall invasion of the tumour by bronchoscopic examination or macroscopic observation during thoracotomy, the patient received a lobectomy without a frozen section examination. Resection was not curative because of the wide submucosal extension to the stump of the resected bronchus. Frozen section examination of the margins of resected bronchus should be considered essential during surgical resection of known adenoid cystic carcinoma, even if the macroscopic appearance is normal.

This case presented with two peripheral lung nodules. In peripherally located "bron- chial adenomas" radiographic findings may be limited to a localised mass unaccompanied by secondary pulmonary changes. ${ }^{8}$ Multiple pulmonary nodules due to peripheral pulmonary adenoid cystic carcinoma has also been reported. ${ }^{6}$ Among the causes of two nodules in this case we considered metastasis from another peripheral pulmonary adenoid cystic carcinoma, two nodules from a peripheral pulmonary adenoid cystic carcinoma, and a concomitant, different disease. Histological examination showed that the peripheral pulmonary adenoid cystic carcinoma was a solitary lung mass, with an associated tuberculoma.

In summary, our case of pulmonary adenoid cystic carcinoma had two uncommon features: the lesion arose in the peripheral lung field, and there was substantial submucosal extension to the proximal bronchial wall.

We thank Drs $T$ Ikeda, $K$ Yagawa, and $N$ Shigematsu fo helpful comments, and Dr Y Miyagawa for preparation of the manuscript. We also thank Mr Brian T Quinn for reviewing the manuscript.

1 de Lima R. Bronchial adenoma: clinicopathologic study and results of treatment. Chest 1980;77:81-4.

2 Spencer H. Pathology of the lung. 4th ed. Oxford: Pergamon, 1985:968-9.

3 Reid JD. Adenoid cystic carcinoma (cylindroma) of the bronchial tree. Cancer 1952;5:685-94.

4 Payne WS, Fontana RS, Woolner LB. Bronchial tumors originating from mucous glands: current classification and unusual manifestations. Med Clin North Am 1968;48: 945-60.

5 Goldstraw P, Lamb D, McCormack RJM, Walbaum PR The malignancy of bronchial adenoma. $J$ Thorac Cardiovasc Surg 1976;72:309-14.

6 Conlan AA, Payne WS, Woolner LB, Sanderson DR. Adenoid cystic carcinoma (cylindroma) and mucoepidermoid carcinoma of the bronchus. $J$ Thorac Cardiovasc Surg 1978;76:369-77.

7 Tolis GA, Fry WA, Head L, Shields TW. Bronchial adenomas. Surg Gynec Obstet 1972;134:605-10.

8 Good CA, and Harrington SW. Asymptomatic bronchial adenoma. Proc Staff Meet Mayo Clin 1953;28:577-86. 